\title{
GOOD KÄHLER METRICS WITH PRESCRIBED SINGULARITIES*
}

\author{
DAMIN WU ${ }^{\dagger}$
}

\begin{abstract}
In this paper, we study the singular Monge-Ampère equations on a quasi-projective manifold with a Poincaré metric. As a consequence, we construct Poincaré Kähler-Einstein metrics which degenerate or grow upward at most like a pole along a given effective divisor.
\end{abstract}

Key words. Quasi-projective manifolds, Singular Monge-Ampère equations, Kähler-Einstein manifolds.

AMS subject classifications. 32Q20, 53C55, 32W20

1. Introduction. Let $\bar{M}$ be a compact Kähler manifold of complex dimension $n$, and $D=\sum_{i=1}^{p} D_{i}$ be a normal crossing divisor, which means that locally $D$ is given by the equation

$$
z^{1} \cdots z^{k}=0, \quad 1 \leq k \leq n,
$$

where $\left(z^{1}, \ldots, z^{n}\right)$ is a local coordinate system on $\bar{M}$. Note that here the irreducible components $D_{i}$ need not be smooth. Locally the complement of $D$ is then given by the polydisks

$$
\left(\Delta^{*}\right)^{k} \times \Delta^{n-k}, \quad 0 \leq k \leq n,
$$

where $\Delta$ is the unit disk in $\mathbb{C}$ and $\Delta^{*}=\Delta \backslash\{0\}$. From now on we denote by

$$
M=\bar{M} \backslash D .
$$

Let $s_{i}$ be the defining section of $D_{i}$, and $|\cdot|$ be a Hermitian metric on the associated line bundle $\left[D_{i}\right]$ such that

$$
\left|s_{i}\right|^{2}<1
$$

on $\bar{M}$, for each $i=1, \ldots, p$. Let $\bar{\omega}$ be a Kähler metric on $\bar{M}$, and let

$$
\omega=C \bar{\omega}-2 \sum_{i=1}^{p} d d^{c} \log \left(-\log \left|s_{i}\right|^{2}\right)
$$

where $C$ is a large constant so that $\omega$ is positive definite on $M$. Here the operator $d^{c}$ is given by

$$
d^{c}=\frac{\sqrt{-1}}{4 \pi}(\bar{\partial}-\partial)
$$

and hence,

$$
d d^{c}=\frac{\sqrt{-1}}{2 \pi} \partial \bar{\partial}
$$

\footnotetext{
*Received January 26, 2008; accepted for publication August 14, 2008

$\dagger$ Department of Mathmatics, The Ohio State University, 1179 University Drive, Newark, OH 43055, USA (dwu@math.ohio-state.edu).
} 
It is easy to check that $\omega$ defines a complete Kähler metric on $M$ of bounded sectional curvature and finite volume. Note that the second term in $\omega$ is dominant near $D$ in the normal direction. Therefore, $\omega$ has Poincaré growth in the normal direction near $D$, and hence, $\omega$ is asymptotic to the product of the classic Poincaré metric and the Euclidean metric

$$
\frac{\sqrt{-1}}{2 \pi} \sum_{i=1}^{k} \frac{d z^{i} \wedge d \bar{z}^{i}}{\left|z^{i}\right|^{2}\left(\log \left|z^{i}\right|^{2}\right)^{2}}+\frac{\sqrt{-1}}{2 \pi} \sum_{j=k+1}^{n} d z^{j} \wedge d \bar{z}^{j}
$$

on the polydisk $\left(\Delta^{*}\right)^{k} \times \Delta^{n-k}$. In particular, $\omega$ induces a good metric, in the sense of Mumford [8], on the logarithmic tangent bundle of $\bar{M}$ along $D$. Similar to [7], we call a metric on $M$ the Poincaré metric associated with $D$, or simply the Poincaré metric, if the metric is uniformly equivalent to $\omega$ and has bounded sectional curvature.

Let $E$ be a closed subset of $\bar{M}$ defined by

$$
E=\{x \in \bar{M} ; \tau(x)=0\},
$$

which is disjoint from the boundary divisor $D$. Here $\tau$ is a smooth nonnegative function on $\bar{M}$ satisfying that

$$
d d^{c} \log (\tau+\epsilon) \geq-C \bar{\omega} \quad \text { for all } 1>\epsilon>0,
$$

where $C$ is a positive constant independent of $\epsilon$, and that $\log \tau \in L^{1}(\bar{M})$, i.e.,

$$
\int_{\bar{M}}|\log \tau| \bar{\omega}^{n}<+\infty
$$

We remark that the $L^{1}$-space on a compact manifold is independent of the metric. Let us fix a Poincaré metric $\omega$ on $M$. Let $\mathcal{R}(M)$ be the Cheng-Yau's Hölder ring (see Section 2 for a definition). Here is our first theorem:

TheOREM 1. Let $E$ and $\tau$ be given as above. For any $F \in \mathcal{R}(M)$ and any positive real number a, there exists a solution $u \in C^{\infty}(M \backslash E)$ of the equations

$$
\begin{aligned}
\left(\omega+d d^{c} u\right)^{n} & =e^{u+F} \tau^{a} \omega^{n}, \\
\omega+d d^{c} u & >0, \quad \text { on } M \backslash E .
\end{aligned}
$$

Furthermore, $u$ is a bounded $C^{1}$-function on $M$ and satisfies that

$$
C^{-1} \tau^{a} \omega \leq \omega+d d^{c} u<C \omega \text { on } M,
$$

where $C$ is a positive constant. In addition, any bounded solution of (1.3) and (1.4), which is smooth outside $E$, must be equal to u plus a constant.

Rather than (1.1), the proof of Theorem 1 need only the weaker inequality

$$
\Delta_{\omega} \log (\tau+\varepsilon)>-C \text { on } M,
$$

where $\Delta_{\omega}$ denotes the negative Laplacian associated with $\omega$. In practice, however, it is sometimes convenient to use following assumption stronger than (1.1):

$$
d d^{c} \log \tau>-C \bar{\omega} \quad \text { on } \bar{M} \backslash E,
$$


and

$$
d d^{c} \tau \geq 0, \quad \text { and } d \tau=0, \quad \text { on } E .
$$

In particular, this setting works for an analytic subvariety which is the intersection of finitely many (possibly singular) hypersurfaces in $\bar{M}$ and which is disjoint from $D$. In fact, let $E_{1}, \ldots, E_{k}$ be the analytic hypersurfaces and $s_{E_{1}}, \ldots, s_{E_{k}}$ be their defining sections (of the induced line bundles), respectively. Then the intersection is given by the zero set of

$$
\tau=\sum_{i=1}^{k}\left|s_{E_{i}}\right|^{2}
$$

where $|\cdot|$ are the metrics on the induced line bundles. It is easy to check that this $\tau$ satisfies (1.5), (1.6), and (1.2).

Next, let us consider the equation with poles on $E$. In this case, $E$ could intersect with $D$, and $\tau$ need not be integrable. But we need to impose certain restriction on the order of the poles. By (1.1) there always exists a positive number $b$, say $1 / 2 C$, such that

$$
\omega+b d d^{c} \log (\tau+\varepsilon)>\lambda \omega
$$

where $\lambda$ is a positive constant independent of $\varepsilon$. Let $b_{+}$be the supremum of all such positive numbers $b$. In particular, $b_{+}=+\infty$ when $\log \tau$ is plurisubharmonic. In the following theorem we require the order $b<b_{+}$, and $b \leq 1$. We remark that, the second assumption $b \leq 1$ can be dropped, in the case that $E$ is an analytic hypersurface contained in the support of $D$.

THEOREM 2. Let $\tau$ be a nonnegative smooth function on $\bar{M}$ and satisfy (1.1), and $E$ is the zero set of $\tau$ in $\bar{M}$. For any $F \in \mathcal{R}(M)$ and any positive real number $b<b_{+}$and $b \leq 1$, there exists a function $u \in C^{\infty}(M \backslash E)$ satisfying

$$
\left(\omega+d d^{c} u\right)^{n}=e^{u+F} \tau^{-b} \omega^{n}
$$

and

$$
C^{-1} \tau^{\Lambda} \omega \leq \omega+d d^{c} u<C \tau^{-\Lambda} \omega
$$

on $M \backslash E$, where $C$ and $\Lambda$ are positive constants. In addition, the solution $u$ has the properties that

$$
\sup _{M \backslash E} u<+\infty, \quad \text { and } \quad \inf _{M \backslash E}(u-b \log \tau)>-\infty \text {. }
$$

We should point out that in the case of compact Kähler manifold, i.e., $M=$ $\bar{M}$, the singular Monge-Ampère equations have been studied comprehensively in the literature, especially in the second part of Yau's celebrated paper [17, p.364-411]. After solving the Calabi's conjecture in the first part of [17], Yau extended his powerful machinery to settle down a very general case of complex Monge-Ampère equation. For instance, the following equation was treated in that paper:

$$
\left(\bar{\omega}+d d^{c} u\right)^{n}=\left(\sum_{i=1}^{p}\left|s_{i}\right|^{2 h_{i}}\right)\left(\sum_{j=1}^{q}\left|t_{j}\right|^{2 k_{j}}\right)^{-1} e^{F(x, u)} \bar{\omega}^{n}
$$


where the $s_{j}$ and $t_{j}$ are, respectively, sections of the holomorphic line bundles $L_{1}$ and $L_{2}$ which satisfy certain mild integrability assumption, and $F$ is a smooth function on $M \times \mathbb{R}$ such that $\partial F / \partial u \geq 0$.

On a complete noncompact manifold, the study of Monge-Ampère equation was initiated by Yau in [18] to understand the Kähler-Einstein metrics, right after his work on the compact case. Following [18], Cheng-Yau [3] solved a large class of nondegerate Monge-Ampère equation on a general complete manifold with positive injectivity radius. They also settle the nondegenerate equation in the case of quasiprojective manifold (see [4] and the references therein). When the right hand side of the equation contains degenerate terms vanishing at $D$ only, the equation was treated by [11] and [19], and by [15] very recently. Note that both [4] and [11] also addressed the orbifold singularities. When the background metric is not Poincaré, certain nondegenerate Monge-Ampère equations were solved by [12] and [13], and also by [1] which assumed the Sobolev inequality.

In contrast to (1.9), at this moment we can only address the simplest singular case on a quasi-projective manifold. Namely, $F(x, u)$ in (1.9) specializes to $u+F(x)$. Also, the zeros and poles have to be treated separately. The major difficulty lies in the fact that most known global or integration methods do not work for the Poincaré metric. In the case of $u+F(x)$, the analysis are local in nature, and cetain maximum principle, such as Yau's upper bound lemma, enables us to obtained the desired estimates.

As for Theorem 1, the point is to refine the upper bound obtained by Yau's upper bound lemma. To derive a uniform upper bound on a compact manifold, one can either use the Moser-Yau's iteration (see, for example, [17]), which depends heavily on the Sobolev inequality, or employ a global positive Green's function (see, for example, $[17$, p.351]). Yet they all break down for the Poincaré metric, due to its finiteness of volume. (See, for example, [6, p.19] and [9, p.378] for the reasons). Motivated by [12] and [9], we overcome this difficulty by using a local positive Dirichlet Green's function. Such an argument need the integrability of $\log \tau$. The uniqueness is proved by using the upper bound, together with Lemma 2, a singular version of the classic Gaffney's theorem.

Under (1.7), the analysis of Theorem 2 is completely local. The equation has meromorphic right hand side. To handle the second order estimate we also need to assume $b \leq 1$, unless $E \subset D$ is a analytic hypersurface. In addition, similar to Theorem 1, we could furthermore derive a uniform lower bound for the solution, by assuming certain integrability of $\tau$. We omit it here, however, since the integrability condition also depends on the bounds of the bisectional curvature of $\omega$. It is unclear whether such a result is useful at this point.

Finally, we consider the Poincaré metric on $M$ which is also Kähler-Einstein. Combining our previous results in [15], we apply Theorem 1 and Theorem 2 to construct certain Kähler-Einstein metrics with singularities. Note that here each irreducible component $D_{i}$ of the normal crossing divisor $D$ is assumed to be nonsingular.

Theorem 3. Let $D=\sum_{i=1}^{p} D_{i}$ be a simple normal crossing divisor in $\bar{M}$. Suppose that there exist real numbers $\alpha_{i} \leq 1$ such that

$$
K_{\bar{M}}+\sum_{i=1}^{p} \alpha_{i}\left[D_{i}\right]>0 \quad \text { on } \bar{M} \text {. }
$$


Assume in addition that

$$
K_{\bar{M}}+\left.[D]\right|_{D_{i}}>0, \quad i=1, \ldots, p
$$

Then, for each effective divisor $E_{Z} \subset \bar{M}, E_{Z} \cap D=\emptyset$, there exists a smooth KählerEinstein metric $\omega_{K E}^{\prime}$ on $M \backslash E$ satisfying that

$$
\begin{gathered}
C^{-1}\left|s_{Z}\right|^{2 \mu} \omega \leq \omega_{K E}^{\prime}<C \omega, \\
C^{-1}\left|s_{Z}\right|^{2 \mu} \omega^{n} \leq\left(\omega_{K E}^{\prime}\right)^{n} \leq C\left|s_{Z}\right|^{2 \mu} \omega^{n},
\end{gathered}
$$

on $M$, where $\omega$ is a Poincaré metric on $M, s_{Z}$ is the defining section of $E_{Z}$, and $C$ and $\mu$ are positive constants. Here one may take $\omega$ to be the unique smooth Poincaré Kähler-Einstein metric on $M$, if one so desires.

We remark that a special useful case of (1.10) and (1.11) is

$$
K_{\bar{M}}+[D]>0 \quad \text { on } \bar{M} .
$$

In some applications, $E_{Z}$ can be viewed as the exceptional divisor obtained from resolving the singularities outside $D$. The singular Poincaré Kähler-Einsein metric $\omega_{K E}^{\prime}$ obtained in Theorem 3 is said to be degenerate at E. Similarly, we can construct a singular Poincaré Kähler-Einstein metrics which may possess a pole.

TheOREM 4. With the same assumption (1.10) and (1.11) as in Theorem 3. Then for any effective divisor $E_{P}$ in $\bar{M}$, there exists a smooth Kähler-Einstein metric $\omega_{K E}^{\prime \prime}$ on $M \backslash E_{P}$ satisfying that

$$
\begin{aligned}
C^{-1}\left|s_{P}\right|^{2 \Lambda} \omega<\omega_{K E}^{\prime \prime} & <C\left|s_{P}\right|^{-2 \Lambda} \omega, \\
C^{-1} \omega^{n}<\left(\omega_{K E}^{\prime \prime}\right)^{n} & \leq C\left|s_{P}\right|^{-2 \mu} \omega^{n},
\end{aligned}
$$

where $\omega$ is a Poincaré metric on $M, s_{P}$ is the defining section of $E_{P}$, and $C, \mu, \Lambda$ are positive constants. If necessary, one may take $\omega$ to be unique nonsingular Poincaré Kähler-Einstein metric on $M$.

In Section 2 we recall the notions of Cheng-Yau's Hölder spaces, and the bounded geometry associated with a Poincaré metric. In Section 3 we collect some tools including their proofs adapted to the geometry. In Section 4 we derive the a priori $C^{0}$ estimates of the perturbed Monge-Ampère equation in Theorem 1 . The $C^{2}$-estimates and uniqueness are obtained in Section 5. In Section 6 we prove Theorem 2. In Section 7 we show Theorem 3 and Theorem 4 by constructing the singular Poincaré Kähler-Einstein metrics.

2. Bounded geometry of quasi-coordinate systems. Let $\bar{M}$ be a compact Kähler manifold, and $D=\sum_{i=1}^{p}$ a normal crossing divisor in $\bar{M}$. We denote by $M$ the complement of $D$ in $\bar{M}$. A Poincaré metric $\omega$ associated with $D$ is given by

$$
\omega=C \bar{\omega}-2 \sum_{i=1}^{p} d d^{c} \log \left(-\log \left|s_{i}\right|^{2}\right),
$$

where $C>0$ is sufficiently large so that $\omega>0$ on $M$. Here each $s_{i}$ is the defining section of $D_{i}$ and $|\cdot|$ is a metric on $\left[D_{i}\right]$. It is clearly that all the Poincaré metrics associated with $D$ are equivalent on $M$. Let us fix a Poincaré metric $\omega$. 
A local coordinate system in $M$ is given by a ploydisk of the form

$$
\left(\Delta^{*}\right)^{k} \times \Delta^{n-k}, \quad 0 \leq k \leq n,
$$

where $\Delta$ and $\Delta^{*}$ are, respectively, the unit disk and the punctured unit disk in the complex plane. The classic Poincaré metric

$$
\omega_{\Delta^{*}}=\frac{\sqrt{-1}}{2 \pi} \frac{d z \wedge d \bar{z}}{|z|^{2}\left(\log |z|^{2}\right)^{2}}
$$

has injectivity radius zero on the punctured disk, so does the Poincaré metric $\omega$ on $M$. Thus when solving some differential equations involving $\omega$, it is more convenient to use the local quasi-coordinate system introduced by Cheng-Yau (see, for example, [14, 800-802] or Section 2 in [15]). The idea is to construct certain nice local holomorphic maps, called quasi-coordinate systems, so that the pullback metric has bounded geometry in the usual sense, i.e., all the derivatives of the pullback metric are bounded.

In the case of $\left(\Delta^{*}, \omega_{\Delta^{*}}\right)$, one can construct the quasi-coordinate systems as follows. Let $\Delta_{3 / 4}$ be the disk centered at the origin with radius $3 / 4$. For each $0<\eta<1$, we define

$$
\phi_{\eta}(v)=\exp \left(\frac{(1+\eta)(v+1)}{(1-\eta)(v-1)}\right), \quad \text { for all } v \in \Delta_{3 / 4} .
$$

It is easy to check that $\phi_{\eta}\left(\Delta_{3 / 4}\right)$ covers $\Delta^{*}$ when $\eta$ runs through $(0,1)$. More importantly, the pullback of the classic Poincaré metric

$$
\phi_{\eta}^{*}\left(\omega_{\Delta^{*}}\right)=\frac{\sqrt{-1}}{2 \pi} \frac{d v \wedge d \bar{v}}{1-|v|^{2}}
$$

is independent of $\eta$, and has bounded geometry on $\Delta_{3 / 4}$ in the usual sense. Hence, $\left\{\left(\Delta_{3 / 4}, \phi_{\eta}\right)\right\}, 0<\eta<1$, are the desired quasi-coordinate systems for $\Delta^{*}$.

For a general quasi-projective manifold $M$, we can similarly construct the quasicoordinate systems. Specifically, for a polydisk with $k$ copies of $\Delta^{*}$, let

$$
V_{\eta}=\left(\Delta_{3 / 4}\right)^{k} \times \Delta^{n-k}, \quad \text { for all } \eta=\left(\eta^{1}, \ldots, \eta^{k}\right) \in(0,1)^{k} .
$$

We define, for each $\eta \in(0,1)^{k}$,

$$
\Phi_{\eta}(v)=\left(\phi_{\eta^{1}}\left(v^{1}\right), \ldots, \phi_{\eta^{k}}\left(v^{k}\right), v^{k+1}, \ldots, v^{n}\right), \quad \text { for all } v \in V_{\eta},
$$

where each $\phi_{\eta^{i}}, 1 \leq i \leq k$, is given by (2.1). Then $\Phi_{\eta}\left(V_{\eta}\right)$ covers the polydisk as $\eta$ runs through $(0,1)^{k}$. The pullback metric $\Phi_{\eta}^{*}(\omega)$ has usual bounded geometry on each $V_{\eta}$. Since $M$ is covered by all the polydisks, we obtain quasi-coordinate systems for $M$ by collecting all the systems $\left\{\left(V_{\eta}, \Phi_{\eta}\right)\right\}$ constructed for the polydisks. We say $(M, \omega)$ has bounded geometry in the sense of quasi-coordinate systems.

For the local analysis on $M$, let us introduce the Cheng-Yau's Hölder spaces which are well adapted to the quasi-coordinate systems on $M$. For a nonnegative integer $k$, and $0<\alpha<1$, one can define the norm $\|\cdot\|_{k, \alpha}$ on $C^{\infty}(M)$ by

$$
\|f\|_{k, \alpha}=\sup _{V_{\eta}}\left\{\left\|\Phi_{\eta}^{*}(f)\right\|_{C^{k, \alpha}\left(V_{\eta}\right)}\right\} .
$$


Then we define the Cheng-Yau's Hölder space $C^{k, \alpha}(M)$ to be the completion of

$$
\left\{f \in C^{\infty}(M) ;\|f\|_{k, \alpha}<+\infty\right\}
$$

with respect to $\|\cdot\|_{k, \alpha}$. It is easy to check that $C^{k, \alpha}(M)$ is a Banach space. In a sense, Cheng-Yau's spaces with respect to a Poincaré metric is similar to the usual Hölder spaces with respect to the standard Euclidean metric. The Cheng-Yau's Hölder ring is defined to be

$$
\mathcal{R}(M)=\bigcap_{k \geq 0,0<\alpha<1} C^{k, \alpha}(M) .
$$

It follows that all the smooth function on $\bar{M}$ belong to $\mathcal{R}(M)$. Besides,

$$
\frac{1}{-\log \left|s_{i}\right|^{2}} \in \mathcal{R}(M)
$$

for each $i=1, \ldots, p$. These functions play an important role in the analysis on $M$ (see [14] and [15]). In this note, however, one only need to bear in mind that $\mathcal{R}(M)$ consists of all the smooth function whose all derivatives are bounded with respect to $\omega$.

For the global analysis on $M$, we define $L^{q}(M, \omega)$ and $W^{l, q}(M, \omega)$, for $1 \leq q \leq+\infty$ and an integer $l \geq 0$, in the usual sense. Namely, $L^{q}(M, \omega)$ consists of all $L^{q}$-integrable functions on $M$ with respect to $\omega . W^{l, q}(M, \omega)$ is the usual Sobolev space with respect to $\omega$ : For each $f \in C^{\infty}(M)$, we define

$$
\|f\|_{W^{l, q}}=\sum_{j=0}^{l}\left\|\nabla^{j} f\right\|_{L^{q}(M, \omega)}
$$

then $W^{l, q}(M, \omega)$ is the completion of

$$
\left\{f \in C^{\infty}(M) ;\|f\|_{W^{l, q}}<+\infty\right\}
$$

with respect to $\|\cdot\|_{W^{l, q}}$.

In this note, we use $C^{k}(M), k \geq 0$, to denote the usual $C^{k}$-function on $M$, while $C^{k, \alpha}(M), k \geq 0$ and $0<\alpha<1$, always denotes the Cheng-Yau's Hölder spaces. By definitions we have, for each $k \geq 0$,

$$
C^{k, \alpha}(M) \subset W^{k, q}(M, \omega), \quad \text { for all } 0<\alpha<1 \text { and all } 1 \leq q \leq+\infty .
$$

And it follows from the usual local Sobolev embedding theorem that

$$
W^{l, q}(M, \omega) \subset C^{m}(M), \quad \text { for all } 0 \leq m<l-\frac{2 n}{q} .
$$

In particular, we will use that a function in $W^{2, \infty}(M, \omega)$ belongs to $C^{1}(M)$.

3. Some useful tools. Maximum principles play an very important role in analysis and geometry. For a complete manifold, we have Yau's generalized maximum principle, and Yau's upper bound lemma. The latter does not even assume the a priori boundedness of the function under consideration. This makes it extremely useful for deriving estimates on complete noncompact manifolds. The following lemma is a 
special case of Yau's upper bound lemma (See, for example, Theorem 8 in [2, p. 353] for a general case).

Lemma 1. Suppose that a function $u \in C^{2}(M)$ satisfies

$$
\Delta_{\omega} u \geq f(u)
$$

where $f$ is a continuous function on $\mathbb{R}$ such that

$$
\liminf _{t \rightarrow+\infty} \frac{f(t)}{t^{\mu}}>0, \quad \text { for some constant } \mu>1 .
$$

Then we have

$$
\sup _{M} u<+\infty, \quad \text { and } \quad f\left(\sup _{M} u\right) \leq 0 .
$$

Let us remark that this lemma still holds when $\omega$ is replaced by any Hermitian metric equivalent to $\omega$. We refer to the Section 3 in [15] for a self-contained short proof. For some applications in this paper, the function $f(t)$ would be the usual exponential function.

The integration method will be used to show the uniqueness of the degenerate equation. The following lemma enables us to integrate by parts. It can essentially be viewed as a special case of Gaffney's theorem ([5, p.141]), which works for an orientable complete Riemannian manifold. The difference here is that the differential forms are allowed to have certain mild singularities. In addition, the proof uses a global distance function associated with $D$. This is similar to the proof of Yau's upper bound lemma in [15]. The global distance function is smooth everywhere, and independent of the Levi-Civita connection. Hence, the proof is simpler and works also for the Hermitian metrics equivalent to $\omega$.

Lemma 2. Let $h \in W^{2,2}(M, \omega)$, and let $\Phi$ be a d-closed real $(n-1, n-1)$-form on $M$, which is smooth outside the zero set of a smooth function on $\bar{M}$, and satisfies that

$$
0 \leq \Phi<C \omega^{n-1} \quad \text { on } M,
$$

for some constant $C>0$. Then we have

$$
\int_{M} d\left(h d^{c} h \wedge \Phi\right)=0 .
$$

As a very special case, let $\Phi=\omega^{n-1}$, and notice that any function in Cheng-Yau's Hölder spaces with $k \geq 2$ belongs to $W^{2,2}(M, \omega)$. Then this lemma implies that

$$
-\int_{M} v d d^{c} v \wedge \omega^{n-1}=\int_{M} d v \wedge d^{c} v \wedge \omega^{n-1},
$$

for all $v \in C^{2, \alpha}(M), 0<\alpha<1$.

Proof of Lemma 2. let $\chi(t)$ be a smooth function which is 0 for $t \leq 0$, between 0 and 1 for $0<t<1$, and 1 for $t \geq 1$. For each $m \geq 1$, we define

$$
\chi_{m}(t)=\chi(t-m) .
$$


Let us denote by

$$
\rho=\sum_{i=1}^{p} \log \left(-\log \left|s_{i}\right|^{2}\right)=\sum_{i=1}^{p} \log \sigma_{i},
$$

where $\sigma_{i}=-\log \left|s_{i}\right|^{2}$. This is the distance function associated with the boundary divisor $D$, and

$$
\rho(x) \rightarrow+\infty
$$

as $x$ approaches $D$. Then

$$
d\left(h d^{c} h \wedge \Phi\right)=d\left[\chi_{m}(\rho) h d^{c} h \wedge \Phi\right]+d\left\{\left[1-\chi_{m}(\rho)\right] h d^{c} h \wedge \Phi\right\} .
$$

Notice that $1-\chi_{m}(\rho)$ has compact support in $M$, and hence the integration of the second term on the right is zero, by the usual Stokes' theorem (together with approximation of smooth forms, if necessary). Therefore,

$$
\int_{M} d\left(h d^{c} h \wedge \Phi\right)=\int_{M} \chi_{m}^{\prime}(\rho) h d \rho \wedge d^{c} h \wedge \Phi+\int_{M} \chi_{m}(\rho) d\left(h d^{c} h \wedge \Phi\right) .
$$

Note that

$$
h d \rho \wedge d^{c} h=\sum_{i=1}^{p} \sigma_{i}^{-1} d \sigma_{i} \wedge h d^{c} h,
$$

and by the Cauchy-Schwarz inequality,

$$
-h^{2} \frac{d \sigma_{i} \wedge d^{c} \sigma_{i}}{\sigma_{i}^{2}}-d h \wedge d^{c} h \leq \frac{d \sigma_{i}}{\sigma_{i}} \wedge h d^{c} h+h d h \wedge \frac{d^{c} \sigma_{i}}{\sigma_{i}} \leq h^{2} \frac{d \sigma_{i} \wedge d^{c} \sigma_{i}}{\sigma_{i}^{2}}+d h \wedge d^{c} h .
$$

Hence,

$$
\left|\frac{\chi_{m}^{\prime}(\rho) h d \rho \wedge d^{c} h \wedge \Phi}{\omega^{n}}\right| \leq \frac{K}{2}\left(h^{2} \sum_{i=1}^{p}\left|\sigma_{i}^{-1} \nabla_{\omega} \sigma_{i}\right|^{2}+C\left|\nabla_{\omega} h\right|^{2}\right),
$$

where $K$ is the upper bound of $\chi^{\prime}(t)$. Then it follows from Lebesgue dominated convergence theorem that

$$
\lim _{m \rightarrow \infty} \int_{M} \chi_{m}^{\prime}(\rho) h d \rho \wedge d^{c} h \wedge \Phi=0 .
$$

Similarly, we also have

$$
\lim _{m \rightarrow \infty} \int_{M} \chi_{m}(\rho) d\left(h d^{c} h \wedge \Phi\right)=0
$$

because of

$$
\left|\frac{\chi_{m}(\rho) d\left(h d^{c} h \wedge \Phi\right)}{\omega^{n}}\right| \leq\left|h \Delta_{\omega} h\right|+C\left|\nabla_{\omega} h\right|^{2},
$$

and by Hölder's inequality $h \Delta_{\omega} h \in L^{1}(M, \omega)$. Thus, we conclude that

$$
\int_{M} d\left(h d^{c} h \wedge \Phi\right)=0 .
$$


4. Degenerate Monge-Ampère equation and zero order estimates. We will use the approximation method to solve the degenerate equation. Let us consider, for $\varepsilon>0$,

$$
\left(\omega+d d^{c} u_{\varepsilon}\right)^{n}=e^{u_{\varepsilon}+F}(\tau+\varepsilon)^{a} \omega^{n} .
$$

Observe that for each $\varepsilon>0$,

$$
\log (\tau+\varepsilon) \in C^{\infty}(\bar{M}) \subset \mathcal{R}(M) .
$$

Therefore, by a well-known result of Cheng-Yau (this can also be viewed as a special case of Lemma 8 in [15] where all $a_{i}=0$.) that there exists a solution $u_{\varepsilon} \in \mathcal{R}(M)$ for (4.1) and the metric defined by

$$
\omega_{\varepsilon}=\omega+d d^{c} u_{\varepsilon}
$$

is equivalent to $\omega$, for each $\varepsilon>0$.

We want to show that there exists a subsequence of $\left\{u_{\varepsilon}\right\}$ which converges to a solution $u \in \mathcal{R}(M)$ of the original degenerate equation. For this purpose, one need to derive a priori uniform Hölder estimates for second order derivatives of $u_{\varepsilon}$, or uniform $C^{3}$-estimates for $u_{\varepsilon}$. For a Monge-Ampère equation like (4.1), the third and higher order estimates can always be localized, as long as one have a priori uniform $C^{2}$-estimates for $u_{\varepsilon}$. The latter depends on the uniform $C^{0}$-estimates of $u_{\varepsilon}$.

In the following, we will always use $C$ to denote a positive constant independent of $\varepsilon$, although it may mean different constants in different inequalities. Notice that for an equation like (4.1), one can always derive a uniform lower bound for $u_{\varepsilon}$ and a uniform upper bound for $u_{\varepsilon}+a \log (\tau+\varepsilon)$. In fact, we have by (4.1)

$$
\begin{aligned}
e^{-u_{\varepsilon}} & =\frac{\left(\omega_{\varepsilon}-d d^{c} u_{\varepsilon}\right)^{n}}{\omega_{\varepsilon}^{n}} e^{F}(\tau+\varepsilon)^{a} \\
& \leq C \frac{\left(\omega_{\varepsilon}-d d^{c} u_{\varepsilon}\right)^{n}}{\omega_{\varepsilon}^{n}} \\
& \leq C\left[1+\frac{1}{n} \Delta^{\prime}\left(-u_{\varepsilon}\right)\right]^{n}
\end{aligned}
$$

where $\Delta^{\prime}$ stands for the negative Laplacian associated with $\omega_{\varepsilon}$. It then follows from Yau's upper bound lemma that

$$
\sup _{M}\left(-u_{\varepsilon}\right) \leq C .
$$

On the other hand, let

$$
v_{\varepsilon}=u_{\varepsilon}+a \log (\tau+\varepsilon) .
$$

Then by (4.1) we have

$$
\begin{aligned}
e^{v_{\varepsilon}} & =e^{-F} \frac{\left[\omega-a d d^{c} \log (\tau+\varepsilon)+d d^{c} v_{\varepsilon}\right]^{n}}{\omega^{n}} \\
& \leq C\left[1-\frac{a}{n} \Delta \log (\tau+\varepsilon)+\frac{1}{n} \Delta v_{\varepsilon}\right]^{n} .
\end{aligned}
$$


Here and from now on, we denote by $\Delta$ the negative Laplacian associated with $\omega$, unless otherwise indicated. By the assumption (1.1),

$$
\Delta \log (\tau+\varepsilon) \geq-C \quad \text { on } M .
$$

Substituting this into (4.3) and applying Yau's upper bound lemma to $v_{\varepsilon}$ yield that

$$
\sup _{M}\left[u_{\varepsilon}+a \log (\tau+\varepsilon)\right]=\sup _{M} v_{\varepsilon} \leq C .
$$

Next, we will improve (4.5) to get a uniform upper bound for $u_{\varepsilon}$, for which we need the integrability of $-\log \tau$ with respect to $\omega$. By the assumption $D \cap E=\emptyset$, there exists a sufficiently small constant $r>0$ such that for each point $p \in E$, the closure of the geodesic ball centered at $p$ with radius $r$, denoted by $\overline{B_{r}(p)}$, is contained in $M$. Clearly $E$ is compact since it is closed in $\bar{M}$. Then there exist finitely many points in $E$, say $p_{1}, \ldots, p_{l}$, such that

$$
E \subset \bigcup_{i=1}^{l} B_{r}\left(p_{i}\right) \equiv E_{r} .
$$

If $u_{\varepsilon}$ achieves or tends to its supremum outside $E_{r}$, then in view of (4.5) we have

$$
\sup _{M} u_{\varepsilon} \leq C+a \sup _{\bar{M} \backslash E_{r}}(-\log \tau),
$$

where the right hand side is independent of $\varepsilon$. Thus we only need to consider $u_{\varepsilon}$ inside $E_{r}$. Without loss of generality, let us assume that

$$
u_{\varepsilon}\left(p_{1}\right)=\sup _{M} u_{\varepsilon} .
$$

Let $G(p, q)$ be the positive Dirichlet Green's function on $B_{r}\left(p_{1}\right)$ (see, for example, [10, p.81]). Namely, $G$ is a nonnegative smooth function defined on the complement of the diagonal in $\overline{B_{r}\left(p_{1}\right)} \times \overline{B_{r}\left(p_{1}\right)}$, and satisfies that

$$
\begin{aligned}
G(p, q) & =G(q, p), \\
\Delta_{q} G(p, q) & =-\delta_{p}(q), \\
G(p, q) & =0, \quad \text { for all } q \in \partial B_{r}\left(p_{1}\right) \text { and } p \neq q,
\end{aligned}
$$

where $\delta_{p}$ is the Dirac $\delta$-function. Furthermore, for small $r$, we have

$$
\begin{aligned}
& |G(p, q)| \leq C(2)|\log d(p, q)|, \quad \text { if } n=1, \\
& |G(p, q)| \leq C(n) d(p, q)^{2-2 n}, \quad \text { if } n \geq 2,
\end{aligned}
$$

and

$$
\left|\nabla_{q} G(p, q)\right| \leq C(n) d(p, q)^{1-2 n} .
$$

Here $d(p, q)$ denotes the geodesic distance between $p$ and $q$, and $C(n)$ are positive constants depending only on $n$. For simplicity, we denote $d(q)=d\left(p_{1}, q\right)$.

Let $\chi(t)$ be a smooth function which is 1 for $t \leq 1 / 2$, between 0 and 1 for $1 / 2<t<3 / 4$, and 0 for $t>3 / 4$. We denote by

$$
\chi_{r}(q)=\chi(d(q) / r), \quad \text { for all } q \in B_{r}\left(p_{1}\right),
$$


where $d(q)$ stands for $d\left(p_{1}, q\right)$. Then $\chi_{r}$ is a cut-off function on $B_{r}\left(p_{1}\right)$ such that $\chi_{r} \equiv 1$ on $B_{r / 2}\left(p_{1}\right)$ and $\chi_{r} \equiv 0$ outside $B_{3 r / 4}\left(p_{1}\right)$. We claim that both $\left|\nabla \chi_{r}\right|$ and $\Delta \chi_{r}$ are bounded independent of $\varepsilon$. Indeed, we have by the construction

$$
\begin{aligned}
\left|\nabla \chi_{r}\right|^{2} & =\left|\chi^{\prime} \nabla d\right|^{2} / r^{2}=\left|\chi^{\prime}\right|^{2} / r^{2}, \\
\Delta \chi_{r} & =\chi^{\prime \prime}|\nabla d|^{2} / r^{2}+\chi^{\prime} \Delta d / r=\chi^{\prime \prime} / r^{2}+\chi^{\prime} \Delta d / r .
\end{aligned}
$$

It follows immediately that $\left|\nabla \chi_{r}\right|$ is uniformly bounded with respect to $\varepsilon$. Since the Poincaré metric $\omega$ has bounded curvature, by the well-known Hessian comparison theorem (see, for example, $[10$, p.5]), we have

$$
\left|\Delta d-\frac{2 n-1}{d}\right| \leq C_{\omega} .
$$

Here the constant $C_{\omega}$ depends only on $n$ and the sectional curvature bounds. On the other hand, $\chi^{\prime}(d(\cdot) / r)$ has compact support in $B_{3 r / 4}\left(p_{1}\right) \backslash B_{r / 2}\left(p_{1}\right)$. Therefore, $\chi^{\prime} \Delta d$ is bounded independently of $\varepsilon$. Hence, $\Delta \chi_{r}$ is also uniformly bounded with respect to $\varepsilon$.

Now we are ready to derive the uniform upper bound for $u_{\varepsilon}$. Since $\omega_{\varepsilon}$ is equivalent to $\omega$, in particular we have

$$
n+\Delta u_{\varepsilon}>0
$$

on $M$. Multiplying both sides by $\chi_{r} G\left(p_{1}, \cdot\right)$ and integrating over $B_{r}\left(p_{1}\right)$ yield that

$$
-\int_{B_{r}\left(p_{1}\right)} \chi_{r}(q) G\left(p_{1}, q\right) \Delta u_{\varepsilon}(q) \omega^{n}(q)<n \int_{B_{r}\left(p_{1}\right)} \chi_{r}(q) G\left(p_{1}, q\right) \omega^{n}(q)
$$

Integrating by parts, it then follows from (4.6) that

$$
\begin{aligned}
u_{\varepsilon}\left(p_{1}\right)< & n \int_{B_{r}\left(p_{1}\right)} \chi_{r}(q) G\left(p_{1}, q\right) \omega^{n}+\int_{B_{r}\left(p_{1}\right)} G\left(p_{1}, q\right) \Delta \chi_{r}(q) u_{\varepsilon}(q) \omega^{n} \\
& +\int_{B_{r}\left(p_{1}\right)}\left|\nabla \chi_{r}(q)\right|\left|\nabla_{q} G\left(p_{1}, q\right)\right|\left|u_{\varepsilon}(q)\right| \omega^{n}(q) .
\end{aligned}
$$

The key observation is that both $\left|\nabla \chi_{r}\right|$ and $\Delta \chi_{r}$ have compact support in $B_{3 r / 4}\left(p_{1}\right) \backslash$ $B_{r / 2}\left(p_{1}\right)$, on which $G\left(p_{1}, q\right)$ and $\left|\nabla_{q} G\left(p_{1}, q\right)\right|$ are both bounded independent of $\varepsilon$, in view of (4.7), (4.8), and (4.9). We have already shown that $\left|\nabla \chi_{r}\right|$ and $\Delta \chi_{r}$ are both uniformly bounded with respect to $\varepsilon$. Hence, we obtain that

$$
u_{\varepsilon}<C+C \int_{M}\left|u_{\varepsilon}\right| \omega^{n} .
$$

Now it follows from (4.3) and our assumption (1.2) that

$$
u_{\varepsilon}<C+a C \int_{M}|\log \tau| \omega^{n}<C_{1},
$$

where $C_{1}$ is positive constant independent of $\varepsilon$. This completes the uniform $C^{0}$ estimates of $u_{\varepsilon}$. 
5. Second order estimates and uniqueness. The uniform $C^{2}$-estimate of $v_{\varepsilon}$ will follows from the standard process, as long as we have the $C^{0}$-estimates, the lower bound of the bisectional curvatures of $\omega$, and (4.4), i.e.,

$$
\Delta \log (\tau+\varepsilon)>-C \quad \text { on } M .
$$

In fact, for any constant $A>0$, we always have

$$
\begin{aligned}
\Delta^{\prime}\left(e^{-A u_{\varepsilon}}\left(n+\Delta u_{\varepsilon}\right)\right) & \geq e^{-A u_{\varepsilon}}\left(n+\Delta u_{\varepsilon}\right)\left(-A \Delta^{\prime} u_{\varepsilon}\right) \\
& +e^{-A u_{\varepsilon}}\left[\Delta^{\prime}\left(\Delta u_{\varepsilon}\right)-\frac{\left|\nabla^{\prime}\left(\Delta u_{\varepsilon}\right)\right|^{2}}{n+\Delta u_{\varepsilon}}\right] .
\end{aligned}
$$

By (4.1), we can estimate as follows the second term on the right of (5.1) using a normal coordinate chart at a point in $M$.

$$
\begin{aligned}
& \Delta^{\prime}\left(\Delta u_{\varepsilon}\right)-\frac{\left|\nabla^{\prime}\left(\Delta u_{\varepsilon}\right)\right|^{2}}{n+\Delta u_{\varepsilon}} \\
& \geq \Delta F-n^{2} \inf _{i \neq j} R_{i \bar{i} \bar{j} \bar{j}}+\Delta \log (\tau+\varepsilon)-n \\
& \quad+\left(n+\Delta u_{\varepsilon}\right)+\left(\inf _{i \neq j} R_{i \bar{i} \bar{j} \bar{j}}\right)\left(n+\Delta u_{\varepsilon}\right) \sum_{j=1}^{n} \frac{1}{1+\left(u_{\varepsilon}\right)_{j \bar{j}}} \\
& \geq-C+\left(n+\Delta u_{\varepsilon}\right)+\inf _{i \neq j} R_{i \bar{i} \bar{j} \bar{j}} \cdot\left(n+\Delta u_{\varepsilon}\right) \sum_{j=1}^{n} \frac{1}{1+\left(u_{\varepsilon}\right)_{j \bar{j}}} .
\end{aligned}
$$

Here (4.4) is used in the last inequality. Observe that the first term on the right of (5.1) can be written as

$$
-A \Delta^{\prime} u_{\varepsilon}=-n A+A \sum_{j=1}^{n} \frac{1}{1+\left(u_{\varepsilon}\right)_{j \bar{j}}} .
$$

Plugging this and (5) into (5.1) yields that

$$
\begin{aligned}
\Delta^{\prime}\left(e^{-A u_{\varepsilon}}\left(n+\Delta u_{\varepsilon}\right)\right) & \geq-C-(n A-1) e^{-A u_{\varepsilon}}\left(n+\Delta u_{\varepsilon}\right) \\
& +\left(A+\inf _{i \neq j} R_{\bar{i} \bar{j} \bar{j}}\right) e^{-A u_{\varepsilon}} \sum_{j=1}^{n} \frac{n+\Delta u_{\varepsilon}}{1+\left(u_{\varepsilon}\right)_{j \bar{j}}},
\end{aligned}
$$

where we use the uniform lower bound of $v_{\varepsilon}$. Notice that

$$
\begin{aligned}
\sum_{j=1}^{n} \frac{1}{1+\left(u_{\varepsilon}\right)_{j \bar{j}}} & \geq \exp \left(-\frac{F+u_{\varepsilon}+\log (\tau+\varepsilon)}{n-1}\right) \cdot\left(n+\Delta u_{\varepsilon}\right)^{\frac{1}{n-1}} \\
& \geq C\left(n+\Delta u_{\varepsilon}\right)^{\frac{1}{n-1}}
\end{aligned}
$$

in view of (4.5). Now let $A$ be sufficiently large so that

$$
A+\inf _{i \neq j} R_{i \bar{i} j \bar{j}}>1 .
$$

Then it follows that

$$
\begin{aligned}
\Delta^{\prime}\left(e^{-A u_{\varepsilon}}\left(n+\Delta u_{\varepsilon}\right)\right) & \geq-C-n A e^{-A u_{\varepsilon}}\left(n+\Delta u_{\varepsilon}\right) \\
& +C_{2}\left(e^{-A u_{\varepsilon}}\left(n+\Delta u_{\varepsilon}\right)\right)^{\frac{n}{n-1}},
\end{aligned}
$$


where $C_{2}$ is a constant independent of $\varepsilon$. Applying Yau's upper bound lemma yields that

$$
e^{-A u_{\varepsilon}}\left(n+\Delta u_{\varepsilon}\right) \leq C .
$$

By $C^{0}$-estimates of $u_{\varepsilon}$ we obtain the desired estimate

$$
n+\Delta u_{\varepsilon} \leq C .
$$

Hence, we have the metric equivalence

$$
C^{-1}(\tau+\varepsilon)^{a} \omega<\omega_{\varepsilon}<C \omega,
$$

which holds uniformly for all $\varepsilon>0$. By this equivalence, one can localize the third and higher order estimates on the relative compact subsets of $M \backslash E$. It then follows from the Ascoli-Arzelà theorem that there exists a subsequence of $\left\{u_{\varepsilon}\right\}$ converging to a solution $u \in C^{\infty}(M \backslash E)$ of (1.3) with

$$
C^{-1} \tau^{a} \omega<\omega+d d^{c} u<C \omega \text { on } M,
$$

as $\varepsilon \rightarrow 0^{+}$. This also implies that $u \in W^{2, \infty}(M, \omega)$; in particular, $u$ is a $C^{1}$-function over $M$. In addition, by (4.2) and (4.11) we have

$$
\sup _{M}|u|<+\infty .
$$

Let us finish Theorem 1 by proving the uniqueness part.

LEMma 3. The bounded solution of (1.3), which is smooth outside $E$ and satisfies (1.4), is unique up to a constant.

Proof. Let $u_{1}$ and $u_{2}$ be two such solutions, and denote by $h=u_{2}-u_{1}$. Then $d d^{c} h$ is bounded with respect to $\omega$, and hence, $h \in W^{2,2}(M, \omega)$. By the local Sobolev embedding we have that $h \in C^{1}(M)$. We would like to show that

$$
d h=0 \quad \text { on } M .
$$

Let $\omega_{i}=\omega+d d^{c} u_{i}, i=1,2$. Then we have by $(1.3)$

$$
\left(\omega_{1}+d d^{c} h\right)^{n}=e^{h} \omega_{1}^{n} .
$$

Subtracting $\omega_{1}^{n}$ from both sides and multiplying by $h$ yield that

$$
h d d^{c} h \wedge\left(\omega_{1}^{n-1}+\omega_{1}^{n-2} \wedge \omega_{2}+\cdots+\omega_{2}^{n-1}\right)=h\left(e^{h}-1\right) \omega_{1}^{n} .
$$

It follows from Lemma 2 that

$$
\int_{M} d\left[h d^{c} h \wedge\left(\omega_{1}^{n-1}+\cdots+\omega_{2}^{n-1}\right)\right]=0 .
$$

Therefore, integrating by parts yields

$$
\begin{aligned}
-\int_{M} h\left(e^{h}-1\right) \omega_{1}^{n} & =\int_{M} d h \wedge d^{c} h \wedge\left(\omega_{1}^{n-1}+\cdots+\omega_{2}^{n-1}\right) \\
& \geq \int_{M} d h \wedge d^{c} h \wedge \omega_{1}^{n-1} .
\end{aligned}
$$


Here the term on the left hand side is nonpositive, in view of the fact that

$$
x\left(e^{x}-1\right) \geq 0, \quad \text { for all } x \in \mathbb{R} .
$$

Then we have

$$
d h=0 \quad \text { on } M \backslash E,
$$

since $\omega_{1}>0$ on $M \backslash E$. Furthermore, $E$ has measure zero since $\log \tau$ is integrable on $\bar{M}$. This together with $h \in C^{1}(M)$ imply (5.3). Hence, we prove that $h$ is a constant on $M$.

6. Singular Monge-Ampère equation. In this section we consider the Monge-Ampère equation with meromorphic right hand side, (1.8). Let us consider its nonsingular perturbation:

$$
\left(\omega+d d^{c} u_{\varepsilon}\right)^{n}=e^{u_{\varepsilon}+F}(\tau+\varepsilon)^{-b} \omega^{n} .
$$

For each $\varepsilon>0$, there exists a $u_{\varepsilon} \in \mathcal{R}(M)$ such that

$$
\omega_{\varepsilon}=\omega+d d^{c} u_{\varepsilon}
$$

is equivalent to $\omega$.

In the following, we use the generic $C$ to denote a positive constant independent of $\varepsilon$. We denote by $\Delta$ and $\Delta^{\prime}$, respectively, the negative Laplacians associated with $\omega$ and $\omega_{\varepsilon}$.

Similar to the degenerate equation, we have

$$
e^{u_{\varepsilon}}=e^{-F}(\tau+\varepsilon)^{b} \frac{\omega_{\varepsilon}^{n}}{\omega^{n}} \leq C\left(1+\frac{1}{n} \Delta u_{\varepsilon}\right)^{n} .
$$

Thus by Yau's upper bound lemma

$$
\sup u_{\varepsilon} \leq C .
$$

Let

$$
v_{\varepsilon}=u_{\varepsilon}-b \log (\tau+\varepsilon) .
$$

By the assumption (1.7), i.e.,

$$
\omega+b d d^{c} \log (\tau+\varepsilon) \geq \lambda \omega, \quad \lambda>0,
$$

we have

$$
\omega_{\varepsilon}=\omega+d d^{c} u_{\varepsilon}>\lambda \omega+d d^{c} v_{\varepsilon} .
$$

Then by $(6.1)$

$$
\begin{aligned}
e^{-v_{\varepsilon}} & =e^{F} \frac{\omega^{n}}{\omega_{\varepsilon}^{n}} \leq e^{\sup F} \lambda^{-n} \frac{\left(\omega_{\varepsilon}-d d^{c} v_{\varepsilon}\right)^{n}}{\omega_{\varepsilon}^{n}} \\
& \leq e^{\sup F} \lambda^{-n}\left(1-\frac{1}{n} \Delta^{\prime} v_{\varepsilon}\right)^{n} .
\end{aligned}
$$


It follows from Yau's upper bound lemma that

$$
\inf v_{\varepsilon}>-C .
$$

Let us now proceed to derive the second order estimate. For any $A>0$, we have

$$
\begin{array}{r}
\Delta^{\prime}\left[e^{-A v_{\varepsilon}}(\tau+\epsilon)\left(n+\Delta u_{\varepsilon}\right)\right] \geq e^{-A v_{\varepsilon}}(\tau+\varepsilon)\left(\Delta^{\prime}\left(\Delta u_{\varepsilon}\right)-\frac{\left|\nabla^{\prime}\left(\Delta u_{\varepsilon}\right)\right|^{2}}{n+\Delta u_{\varepsilon}}\right) \\
+e^{-A v_{\varepsilon}}(\tau+\varepsilon)\left(n+\Delta u_{\varepsilon}\right)\left[-A \Delta^{\prime} v_{\varepsilon}+\Delta^{\prime} \log (\tau+\varepsilon)\right] .
\end{array}
$$

By (6.1), we estimate in a normal coordinate system of a point:

$$
\begin{aligned}
(\tau+\varepsilon)\left(\Delta^{\prime}\left(\Delta u_{\varepsilon}\right)-\right. & \left.\frac{\left|\nabla^{\prime}\left(\Delta u_{\varepsilon}\right)\right|^{2}}{n+\Delta u_{\varepsilon}}\right) \geq(\tau+\varepsilon)\left(\Delta F-n-n^{2} \inf _{i \neq l} R_{i \bar{i} \bar{l} \bar{l}}\right) \\
& -b(\tau+\varepsilon) \Delta \log (\tau+\varepsilon)+(\tau+\varepsilon)\left(n+\Delta u_{\varepsilon}\right) \\
& +(\tau+\varepsilon)\left(n+\Delta u_{\varepsilon}\right) \inf _{i \neq l} R_{i \bar{i} l \bar{l}} \sum_{i} \frac{1}{1+\left(u_{\varepsilon}\right)_{i \bar{i}}} .
\end{aligned}
$$

Observe that

$$
(\tau+\epsilon) \Delta \log (\tau+\varepsilon)=\Delta \tau-\frac{|\nabla \tau|^{2}}{\tau+\varepsilon} \leq \Delta \tau \leq C .
$$

Thus, we have

$$
\begin{gathered}
(\tau+\varepsilon)\left(\Delta^{\prime}\left(\Delta u_{\varepsilon}\right)-\frac{\left|\nabla^{\prime}\left(\Delta u_{\varepsilon}\right)\right|^{2}}{n+\Delta u_{\varepsilon}}\right) \geq-C+(\tau+\varepsilon)\left(n+\Delta u_{\varepsilon}\right) \\
+(\tau+\varepsilon)\left(n+\Delta u_{\varepsilon}\right) \inf _{i \neq l} R_{i \bar{i} l \bar{l}} \sum_{i} \frac{1}{1+\left(u_{\varepsilon}\right)_{i \bar{i}}} .
\end{gathered}
$$

On the other hand, by the assumption (1.7) we have

$$
\begin{aligned}
-d d^{c} v_{\varepsilon} & =-\omega-d d^{c} u_{\varepsilon}+\left[\omega+b d d^{c} \log (\tau+\varepsilon)\right] \\
& \geq-\omega_{\varepsilon}+\lambda \omega .
\end{aligned}
$$

Thus,

$$
-\Delta^{\prime} v_{\varepsilon} \geq \lambda \sum_{i} \frac{1}{1+\left(u_{\varepsilon}\right)_{i \bar{i}}}-n
$$

Similarly, by (1.7) or (1.1)

$$
\Delta^{\prime} \log (\tau+\varepsilon) \geq-C \sum_{i} \frac{1}{1+\left(u_{\varepsilon}\right)_{i \bar{i}}} .
$$

Hence,

$$
\begin{gathered}
(\tau+\varepsilon)\left(n+\Delta u_{\varepsilon}\right)\left[-A \Delta^{\prime} v_{\varepsilon}+\Delta^{\prime} \log (\tau+\varepsilon)\right] \geq-n(\tau+\varepsilon)\left(n+\Delta u_{\varepsilon}\right) \\
+(\tau+\varepsilon)\left(n+\Delta u_{\varepsilon}\right)(A \lambda-C) \sum_{i} \frac{1}{1+\left(u_{\varepsilon}\right)_{i \bar{i}}}
\end{gathered}
$$


Now let $A$ be sufficiently large such that

$$
A \lambda-C+\inf _{i \neq l} R_{i \bar{i} l \bar{l}}>1
$$

Then plugging (6.5) and (6.6) into (6.4) yields that

$$
\begin{aligned}
\Delta^{\prime}\left[e^{-A v_{\varepsilon}}(\tau+\epsilon)\left(n+\Delta u_{\varepsilon}\right)\right] & \geq-C-(A n-1) e^{-A v_{\varepsilon}}(\tau+\varepsilon)\left(n+\Delta u_{\varepsilon}\right) \\
& +e^{-A v_{\varepsilon}}(\tau+\varepsilon)\left(n+\Delta u_{\varepsilon}\right) \sum_{i} \frac{1}{1+\left(u_{\varepsilon}\right)_{i \bar{i}}} .
\end{aligned}
$$

Notice that

$$
\begin{aligned}
\sum_{i} \frac{1}{1+\left(u_{\varepsilon}\right)_{i \bar{i}}} & \geq \exp \left(-\frac{u_{\varepsilon}+F-b \log (\tau+\varepsilon)}{n-1}\right)\left(n+\Delta u_{\varepsilon}\right)^{\frac{1}{n-1}} \\
& \geq C(\tau+\varepsilon)^{\frac{b}{n-1}}\left(n+\Delta u_{\varepsilon}\right)^{\frac{1}{n-1}}
\end{aligned}
$$

Therefore, we obtain

$$
\begin{gathered}
\Delta^{\prime}\left[e^{-A v_{\varepsilon}}(\tau+\epsilon)\left(n+\Delta u_{\varepsilon}\right)\right] \geq-C-(A n-1) e^{-A v_{\varepsilon}}(\tau+\varepsilon)\left(n+\Delta u_{\varepsilon}\right) \\
+C_{3}(\tau+\varepsilon)^{\frac{b-1}{n-1}}\left[e^{-A v_{\varepsilon}}(\tau+\varepsilon)\left(n+\Delta u_{\varepsilon}\right)\right]^{\frac{n}{n-1}},
\end{gathered}
$$

where $C_{3}$ is a positive constant independent of $\varepsilon$. Because of the assumption $b \leq 1$, the term $(\tau+\varepsilon)^{b-1}$ has uniform lower bound on $M$. Therefore, we can apply Yau's upper bound lemma to get that

$$
e^{-A v_{\varepsilon}}(\tau+\varepsilon)\left(n+\Delta u_{\varepsilon}\right) \leq C .
$$

Consequently,

$$
(\tau+\varepsilon)^{1+A b}\left(n+\Delta u_{\varepsilon}\right) \leq C e^{A u_{\varepsilon}} \leq C .
$$

This implies that $n+\Delta u_{\varepsilon}$ is uniformly bounded over relative compact subsets in $M \backslash E$. Then, one can get the uniform third order estimates on the subsets. Therefore, there exists a subsequence of $\left\{u_{\varepsilon}\right\}$ converging to a solution $u \in C^{\infty}(M \backslash E)$ of

$$
\begin{aligned}
\left(\omega+d d^{c} u\right)^{n} & =e^{u+F} \tau^{-b} \omega^{n} \\
C^{-1} \tau^{\Lambda} \omega & <\omega+d d^{c} u<C \tau^{-\Lambda} \omega, \quad C>0, \Lambda>0,
\end{aligned}
$$

on $M \backslash E$, as $\varepsilon$ tends to zero. In addition, by (6.2) and (6.3), we obtain

$$
\sup _{M \backslash E} u<+\infty, \quad \text { and } \quad \inf _{M \backslash E}(u-b \log \tau)>-\infty .
$$

Let us remark that, in the case that $E$ is an analytic hypersurface contained in the support of $D$, i.e., $\tau=\prod_{i=1}^{p}\left|s_{i}\right|^{2 \alpha_{i}}$ for each $\alpha_{i} \geq 0$. Then we can drop the assumption that $b \leq 1$ in Theorem 2. In fact, in this case one should replace the factor $(\tau+\varepsilon)$ by $\tau$ in (6.4), and apply the usual maximum principle (see, for example, Section 4 in $[15])$. 
7. Applications to Kähler-Einstein metrics. In this section, we consider the Poincaré metric which is also Einstein. In this paper, we call such a metric a Poincaré Kähler-Einstein metric. Notice that a Poincaré Kähler-Einstein metric must be of negative scalar curvature, because of its finiteness of volume (by [16, p.667] a complete noncompact Ricci-flat Kähler manifold always has infinite volume.).

Recently in [15], we have investigated certain necessary and sufficient conditions for the existence of a Poincaré Kähler-Einstein metric on $M$. Specifically, we assume that there exist real numbers $\alpha_{i} \leq 1, i=1, \ldots, p$, such that

$$
K_{\bar{M}}+\sum_{i=1}^{p} \alpha_{i}\left[D_{i}\right]>0 \quad \text { on } \bar{M} .
$$

This assumption is conjectured to be the necessary condition for the existence of a (possibly incomplete) Kähler-Einstein metric on $M$ with negative curvature. Let us also assume that each irreducible component $D_{i}$ is smooth; i.e., $D=\sum_{i} D_{i}$ is a simple normal crossing divisor. Furthermore, if

$$
\left.\left(K_{\bar{M}}+[D]\right)\right|_{D_{i}}>0
$$

for each $D_{i}$, then $M$ admits a unique Poincaré Kähler-Einstein metric.

Now suppose that $M$ possesses a Poincaré Kähler-Einstein metric $\omega_{K E}$. Let $E_{Z}$ be an effective divisor in $\bar{M}$. First, we would like to construct a Kähler-Einstein metric $\omega_{K E}^{\prime}$ on $M \backslash E_{Z}$, which has Poincaré growth near $D$ and degenerates at $E_{Z}$ in the following sense. We call such a metric a Poincaré Kähler-Einstein metric degenerate at $E_{Z}$, or simply degenerate Poincaré Kähler-Einstein metric.

For this purpose, let us assume that $E_{Z}$ is disjoint from $D$. This is possible, even for $D$ to be a normal crossing divisor. For instance, let $E_{Z}$ be the exceptional divisor obtained from blowing up a subvariety in the complement of $D$ in $\bar{M}$. For an example which is not a blowing up, let $\bar{M}$ be an algebraic surface which has fibration over a curve; then let $D$ and $E_{Z}$ be different fibers, whose multiplicities could be greater than one.

Let $h_{Z}$ be a metric on $\left[E_{Z}\right]$ and $s_{Z}$ be the defining section of $E_{Z}$. Since $\omega_{K E}$ is a Poincaré metric, we can choose a sufficiently small real number $\mu>0$ so that

$$
\omega_{K E}+\mu d d^{c} \log h_{Z}>0 \text { on } M .
$$

Let us fix such a $\mu$. Then

$$
\omega_{\mu}=\omega_{K E}+\mu d d^{c} \log h_{Z}
$$

is a Poincaré metric on $M$.

Let

$$
F=\log \left(\frac{\omega_{K E}^{n}}{\omega_{\mu}^{n}}\right) .
$$

Then $F \in \mathcal{R}(M)$. Thus, we arrive at the following Monge-Ampère equation

$$
\left(\omega_{\mu}+d d^{c} u\right)^{n}=\omega_{\mu}^{n} e^{u+F}\left|s_{Z}\right|^{2 \mu} .
$$

Clearly, here $\tau=\left|s_{Z}\right|^{2}$ satisfies the conditions (1.5), (1.6), and (1.2). It then follows from Theorem 1 that there exists a solution $u$ of $(7.2)$ so that

$$
\omega_{K E}^{\prime}=\omega_{\mu}+d d^{c} u
$$


is the desired Poincaré metric which is Einstein on $M \backslash E$ and degenerates exactly at $E$ :

$$
C^{-1}\left|s_{Z}\right|^{2 \mu} \omega_{K E}<\omega_{K E}^{\prime}<C \omega_{K E} \quad \text { on } M,
$$

where $C>0$ is a constant. Furthermore, by (7.2) we obtained that

$$
C^{-1}\left|s_{Z}\right|^{2 \mu}\left(\omega_{K E}\right)^{n} \leq\left(\omega_{K E}^{\prime}\right)^{n} \leq C\left|s_{Z}\right|^{2 \mu}\left(\omega_{K E}\right)^{n} \quad \text { on } M,
$$

for some constant $C>0$. This proves Theorem 3 .

Let us consider the case that $D \cap E \neq \emptyset$. It would be interesting to see whether one can construct a Poincaré metric, which is Einstein and degenerates at $E$ outside a tubular neighborhood of $D$. One possible way is to apply the Theorem 1 . At this moment, it is unclear how to construct an appropriate function $\tau$ which satisfies (1.1).

Finally, let us construct a Poincaré Kähler-Einstein metric which possesses a pole near an effective divisor $E_{P}$ in $\bar{M}$. Suppose that $M$ admits a Poincaré Kähler-Einstein metric. Let $h_{P}$ be a metric on $\left[E_{P}\right]$ and $s_{P}$ be the defining section of $E_{P}$. Similar to the degenerate case, let us choose a sufficiently small positive real number $\nu$ such that

$$
\omega_{K E}-\nu d d^{c} \log h_{P}>0 .
$$

Let us fix such a $\nu$, and then

$$
\omega_{\nu}=\omega_{K E}-\nu d d^{c} \log h_{P}
$$

defines a Poincaré metric on $M$. We denote by

$$
F=\log \left(\frac{\omega_{K E}^{n}}{\omega_{\nu}^{n}}\right) .
$$

Then $F \in \mathcal{R}(M)$. Let us consider the following singular Monge-Ampère equation

$$
\left(\omega_{\nu}+d d^{c} u\right)^{n}=e^{u+F}\left|s_{P}\right|^{-2 \nu} \omega_{\nu}^{n} \quad \text { on } M \backslash E_{P} .
$$

By Theorem 2 there exists a function $u \in C^{\infty}\left(M \backslash E_{P}\right)$ satisfying (7.3) with $\sup _{M} u<$ $+\infty$. Thus, by construction,

$$
\omega_{K E}^{\prime \prime}=\omega_{\nu}+d d^{c} u
$$

defines a smooth Kähler-Einstein metric on $M \backslash E$, and $\left(\omega_{K E}^{\prime \prime}\right)^{n}(x)$ grows upward at most like $\left|s_{P}\right|^{-2 \nu} \omega_{K E}^{n}$ when $x$ approaches $E$. More precisely, we have on $M \backslash E$ that

$$
\begin{aligned}
& C^{-1}\left|s_{P}\right|^{2 \Lambda} \omega_{K E}<\omega_{K E}^{\prime \prime}<C\left|s_{P}\right|^{-2 \Lambda} \omega_{K E}, \\
& C^{-1}\left(\omega_{K E}\right)^{n}<\left(\omega_{K E}^{\prime \prime}\right)^{n} \leq C\left|s_{P}\right|^{-2 \mu}\left(\omega_{K E}\right)^{n},
\end{aligned}
$$

where $C, \mu, \Lambda$ are all positive constants. This completes the proof of Theorem 4 .

Acknowledgements. First of all, the author would like to thank Professor Yau for his help and his mathematical influence. The author would also like to thank Professor F. Zheng for discussions, especially for providing the examples on the disjointedness of the divisors in the last section. The author would like to thank Professors D. Burns, P. Li, D. Phong, and R. Schoen for their interests and discussions. Finally, he would also thank the referee for the careful proofreading. 


\section{REFERENCES}

[1] S. Bando AND R. KoBAYAhsi, Ricci-flat Kähler metrics on affine algebraic manifolds II, Math. Ann., 287 (1990), pp. 175-180.

[2] S. Y. Cheng and S.-T. Yau, Differential equations on Riemannian manifolds and their gometric applications, Comm. Pure Appl. Math., 28 (1975), pp. 333-354.

[3] S. Y. Cheng And S.-T. YAu, On the existence of a complete Kähler metric on non-compact complex manifolds and the regularity of Fefferman's equation, Comm. Pure Appl. Math., 33 (1980), pp. 507-544.

[4] S. Y. CHENG AND S.-T. YAU, Inequality between Chern numbers of singular Kähler surfaces and characterization of orbit space of discrete group of $S U(2,1)$, Contemporary Mathematics, 49 (1986), pp. 31-43.

[5] M. Gaffney, A special Stokes's thoerem for complete Riemannian manifolds, Ann. of Math., 60 (1954), pp. 140-145.

[6] E. Hebey, Sobolev spaces on Riemannian manifolds, Lecture Notes in Mathematics, 1635, Springer-Verlag, Berlin, 1996.

[7] Z. Lu And X. Sun, On the Weil-Petersson volume and the first Chern class of the Moduli space of Calabi-Yau manifolds, Commun. Math. Phys., 261 (2006), pp. 297-322.

[8] D. Mumford, Hirzebruch's proportionality theorem in the non-compact case, Invent. Math., 42 (1977), pp. 239-272.

[9] P. LI, Curvature and function theory on Riemannian manifolds, In: Surveys in differential Geometry VII, International Press, Somerville, MA, 2000, pp. 375-432.

[10] R. Schoen and S.-T. Yau, Lectures on Differential Geometry, International Press, 1994.

[11] G. TIAn AND S.-T. YAU, Existence of Kähler-Einstein metrics on complete Kähler manifolds and their applications to algebraic geometry, Proc. Conf. San Diego. Adv. Ser. Math. Phys., 1 (1987), pp. 574-629.

[12] G. Tian and S.-T. YAU, Complete Kähler manifolds with zero Ricci curvature. I, J. Amer. Math. Soc., 3 (1990), pp. 579-609.

[13] G. Tian And S.-T. Yau, Complete Kähler manifolds with zero Ricci curvature. II, Invent. Math., 106 (1991), pp. 27-60.

[14] D. Wu, Higher canonical asymptotics of Kähler-Einstein metrics on quasi-projective manifolds, Comm. Anal. Geom., 14:4 (2006), pp. 795-845.

[15] D. Wu, Kähler-Einstein metrics of negative Ricci curvature on general quasi-projective manifolds, submitted to Comm. Anal. Geom.

[16] S.-T. YAU, On some function theoretic properties of a complete Riemannian manifold and their applications in geometry, Indiana Univ. Math. J., 25 (1976), pp. 659-670.

[17] S.-T. YAU, On the Ricci curvature of a compact Kähler manifold and the complex MongeAmpère equation I, Comm. Pure Appl. Math., 31 (1978), pp. 339-411.

[18] S.-T. YAU, Métriques de Kähler-Einstein sur les variétés ouvertes, in Première Classe de Chern et courbure de Ricci: Preuve de la conjecture de Calabi, Séminaire Palaiseau, Astérisque, 58 (1978), pp. 163-167.

[19] S.-T. YAU, A splitting theorem and algebraic geometric characterization of locally Hermitian symmetric spaces. Comm. Anal. Geom., 1:3 (1993), pp. 473-486. 